\title{
A GENERALIZATION OF UNIVALENT FUNCTIONS WITH BOUNDED BOUNDARY ROTATION
}

\author{
BY
}

EDWARD J. MOULIS, JR.(1)

ABSTRACT. This paper introduces a class of functions which generalizes both those functions $f(z)$ with bounded boundary rotation and those functions for which $z f^{\prime}(z)$ is $a$-spirallike. A simple variational formula for this class is derived and used to determine sufficient conditions for the univalency of functions there in. Various representations for these functions are given, and these are used to derive another condition for univalence; this one is the best known so far in the subclass consisting of functions $f(z)$ for which $z f^{\prime}(z)$ is $\alpha$-spirallike. Bounds on the modulus of the Schwarzian derivative are also derived; these are sharp in the subclass of functions having bounded boundary rotation.

1. Introduction. Let $Q_{a}^{k}$ denote the class of functions $q(z)$ having the properties that $q(z)$ is regular in $E=\{z:|z|<1\}, q(0)=1$, and, for every $r, 0 \leq r<1$,

$$
\left.\int_{0}^{2 \pi} \mid \operatorname{Re}\left\{e^{i a} q\left(r e^{i \theta}\right)\right)\right\} \mid d \theta \leq k \pi \cos \alpha,
$$

where $k \geq 2$ and $\alpha$ is real, $|\alpha|<\pi / 2$. Recently, M. S. Robertson [11] derived a variational formula for this class of functions. The class $Q_{0}^{2}$ is the well-known class $P$ consisting of normalized functions which map $E$ onto the right half-plane. In this paper, we introduce and study a related class of functions.

$V_{\alpha}^{k}$ will denote the class of functions $f(z)$ which are regular in $E$, satisfy $f(0)=0, f^{\prime}(0)=1$ and $f^{\prime}(z) \neq 0$ in $E$, and have the property that $1+z f^{\prime \prime}(z) / f^{\prime}(z)$ is in $Q_{a}^{k}$. Hence functions in $V_{a}^{k}$ satisfy the condition

$$
\int_{0}^{2 \pi}\left|\operatorname{Re}\left\{e^{i \alpha}\left[1+\frac{z f^{\prime \prime}(z)}{f^{\prime}(z)}\right]\right\}\right| d \theta \leq k \pi \cos \alpha, \quad 0 \leq r<1, z=r e^{i \theta},
$$

where $k$ and $\alpha$ are real constants satisfying the same constraints as above.

Functions in $V_{0}^{k}$ have bounded boundary rotation, and were first studied by Lowner [5] and Paatero ([7], [8]). Functions in the class $V_{\alpha}^{k}$ with $\alpha \neq 0$ no longer necessarily have bounded boundary rotation, but they possess other interesting properties. Since the integrand in (1.1) is the absolute value of a function harmonic in $E$, functions in $V_{\alpha}^{2}$ satisfy

Received by the editors December 1, 1971.

AMS (MOS) subject classifications (1970). Primary 30A32; Secondary 30A32.

Key word and phrases. Univalent functions, boundary rotation, a-spirillike functions, Schwarzian derivative.

(1) Portions of this paper comprise a doctoral dissertation written at the University of Delaware under the direction of Professor Malcolm S. Robertson. 


$$
\int_{0}^{2 \pi}\left|\operatorname{Re}\left\{e^{i \alpha}\left[1+\frac{z f^{\prime \prime}(z)}{f^{\prime}(z)}\right]\right\}\right| d \theta=2 \pi \cos \alpha, \quad z=r e^{i \theta}, 0 \leq r<1 .
$$

An argument based on the continuity of the integrand in (1.2) shows that we must have

$$
\operatorname{Re}\left\{e^{i a}\left[1+\frac{z f^{\prime \prime}(z)}{f^{\prime}(z)}\right]\right\}=\operatorname{Re}\left\{e^{i a}\left[z \frac{\left[z f^{\prime}(z)\right]^{\prime}}{z f^{\prime}(z)}\right]\right\}>0, \quad z \text { in } E, f(z) \text { in } V_{a}^{2}
$$

which means that $z f^{\prime}(z)$ is $\alpha$-spirallike in $E$.

Paatero [7] showed that functions in $V_{0}^{k}$ are univalent whenever $2 \leq k \leq 4$. Recently, Robertson [10] showed that functions in $V_{a}^{2}$ are univalent for a certain range of $\cos \alpha$. This paper gives various conditions on $k$ and $\alpha$ which assure that functions in $V_{a}^{k}$ are univalent. The techniques used to obtain the se conditions also provide insight into other analytic and geometric properties of $V_{a}^{k}$.

2. Representation theorems and a coefficient bound. We first derive a representation for the class $Q_{a}^{k}$ with the help of a theorem found in [7].

Theorem. Let $f(z)=u(z)+i v(z)$ be regular in $E$. If $z=r e^{i \phi}$ and $\int_{0}^{2}\left|u\left(r e^{i \phi}\right)\right| d \phi$ is bounded for $r<1$, there exists a real-valued function $\Psi(\theta)$ with bounded variation in $[0,2 \pi]$ such that, for $z$ in $E$,

$$
f(z)=\frac{1}{2 \pi} \int_{0}^{2 \pi} \frac{1+z e^{i \theta}}{1-z e^{i \theta}} d \psi(\theta)+i v(0) .
$$

The total variation of $\psi(\theta)$ is given by

$$
\int_{0}^{2 \pi}|d \psi(\theta)|=\lim _{r \rightarrow 1} \int_{0}^{2 \pi}\left|u\left(r e^{i \phi}\right)\right| d \phi .
$$

If we set $f(z)=\left(e^{i a} q(z)-i \sin \alpha\right) / \cos \alpha$, then $u(z)=\operatorname{Re}\{f(z)\}=\operatorname{Re}\left\{e^{i a} q(z) / \cos \alpha\right\}$. If $q(z)$ belongs to $Q_{a}^{k}$, then

$$
\int_{0}^{2 \pi}\left|\operatorname{Re}\left\{\frac{e^{i \alpha} q(z)}{\cos \alpha}\right\}\right| \leq k \pi, \quad z=r e^{i \theta}, 0 \leq r<1
$$

Further,

$$
\begin{aligned}
& v(z)=\operatorname{Im}\{f(z)\}=\operatorname{Im}\left\{e^{i \alpha} q(z) / \cos \alpha\right\}-(\sin \alpha) / \cos \alpha, \\
& v(z)=\tan \alpha \operatorname{Re}\{q(z)\}+\operatorname{Im} q(z)-\tan \alpha, \\
& v(0)=0
\end{aligned}
$$

$f(z)$ is regular in $|z|<1$ for $|a|<\pi / 2$, so Paatero's theorem gives the following representation.

Theorem 1. If $q(z)$ is in $Q_{a}^{k}$, then

$$
e^{i a} q(z)=\frac{\cos \alpha}{2 \pi} \int_{0}^{2 \pi} \frac{1+z e^{i \phi}}{1-z e^{i \phi}} d \dot{\mu}(\phi)+i \sin \alpha, \quad|z|<1,
$$


where $\psi(\phi)$ is a function with bounded variation on $[0,2 \pi]$ satisfying

$$
\int_{0}^{2 \pi} d \psi(\phi)=2 \pi
$$

and

$$
\int_{0}^{2 \pi}|d \psi(\phi)|=\lim _{r \rightarrow 1} \int_{0}^{2 \pi}\left|\operatorname{Re}\left\{\frac{e^{i \alpha} q(z)}{\cos \alpha}\right\}\right| d \theta \leq k \pi
$$

We use this theorem and another representation theorem due to Paatero [ 7 ] which states that functions $f_{0}(z)$ in $V_{0}^{k}$ may be written

$$
f_{0}(z)=\int_{0}^{z} \exp \left(-\frac{1}{\pi} \int_{0}^{2 \pi} \log \left(1-z e^{i \theta}\right) d \psi(\theta)\right) d z
$$

where $\psi(\theta)$ satisfies conditions (2.2) and (2.3). Differentiation of both sides of (2.4) yields

$$
1+\frac{z f_{0}^{\prime \prime}(z)}{f_{0}^{\prime}(z)}=\frac{1}{2 \pi} \int_{0}^{2 \pi} \frac{1+z e^{i \theta}}{1-z e^{i \theta}} d \psi(\theta),
$$

and so substitution in (2.1) gives

$$
e^{i a}\left[1+\frac{z f_{a}^{\prime \prime}(z)}{f_{a}^{\prime}(z)}\right]=(\cos \alpha)\left[1+\frac{z f_{0}^{\prime \prime}(z)}{f_{0}^{\prime}(z)}\right]+i \sin \alpha,
$$

where $f_{a}(z)$ is an appropriate function in $V_{a}^{k}$. Solving this differential equation yields the next theorem.

Theorem 3. $f_{a}(z)$ belongs to $V_{a}^{k}$ if and only if there is a function $f_{0}(z)$ in $V_{0}^{k}$ sucb that

$$
f_{\alpha}^{\prime}(z)=\left[f_{0}^{\prime}(z)\right]^{e^{-i \alpha}} \cos \alpha
$$

Corollary. $f_{a}(z)$ belongs to $V_{a}^{k}$ if and only if

$$
f_{\alpha}(z)=\int_{0}^{z} \exp \left(-\frac{e^{-i \alpha} \cos \alpha}{\pi} \int_{0}^{2 \pi} \log \left(1-z e^{i \theta}\right) d \psi(\theta)\right) d z
$$

where $\psi(\theta)$ satisfies (2.2) and (2.3).

Theorem 4. $f_{\alpha}(z)$ belongs to $V_{a}^{k}$ if and only if there are two normalized starlike functions $S_{1}(z)$ and $S_{2}(z)$ such that

$$
f_{\alpha}^{\prime}(z)=\left\{\frac{\left[S_{1}(z) / z\right]^{(k+2) / 4}}{\left[S_{2}(z) / z\right]^{(k-2) / 4}}\right\} e^{-i \alpha} \cos a
$$

Proof. According to Brannan [2], $f_{0} \in V_{0}^{k}$ if and only if

$$
f_{0}^{\prime}(z)=\left[S_{1}(z) / z\right]^{(k+2) / 4} /\left[S_{2}(z) / z\right]^{(k-2) / 4},
$$


where $S_{1}(z)$ and $S_{2}(z)$ are normalized starlike functions. Theorem 4 follows an application of Theorem 3 to (2.7).

Corollary. $f(z)$ is in $V_{\alpha}^{k}$ if and only if there exist two normalized a-spiral functions $T_{1}(z)$ and $T_{2}(z)$ such that

$$
f^{\prime}(z)=\left[T_{1}(z) / z\right]^{(k+2) / 4} /\left[T_{2}(z) / z\right]^{(k-2) / 4} \text {. }
$$

Proof. If $S(z)$ is starlike, then $T(z)=z[S(z) / z]^{e^{-i a} \cos \alpha}$ is a-spirallike, since $z T^{\prime}(z) / T(z)=e^{-i a}(\cos \alpha) z S^{\prime}(z) / S(z)$.

Theorem 5. If $f(z)=z+a_{2} z^{2}+\cdots$ is in $V_{a}^{k}$, then $\left|a_{2}\right| \leq(k / 2) \cos \alpha$. This bound is sharp.

Proof. Differentiate both sides of (2.6) and use the bound on $\left|a_{2}\right|$ derived by Pick for functions in $V_{0}^{k}$ (see Lehto [4]). To see that there is a function with a second coefficient having a modulus of $(k / 2) \cos \alpha$, recall that Paatero has shown [7] that the function

$$
f_{0}(z)=\frac{1}{k}\left[\left(\frac{1+z}{1-z}\right)^{k / 2}-1\right]
$$

is in $V_{0}^{k}$. By Theorem 3,

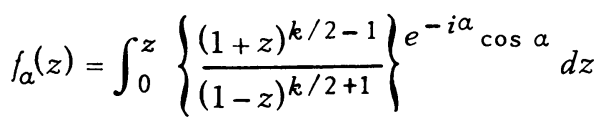

is in $V_{a}^{k}$, and $f_{a}^{\prime \prime}(0)=k e^{-i \alpha} \cos \alpha$.

3. A simple test for univalency in $V_{a}^{k}$. We now consider a variational formula used by Ziegler [12] whose generalization of close-to-convex functions included the class $V_{a}^{2}$. We now show that this formula is valid for $k>2$.

Theorem 6. If $f(z)$ belongs to $V_{a}^{k}$ and if $F(z)$ is defined by

$$
F^{\prime}(z)=f^{\prime}\left(\frac{z+a}{1+a z}\right) / f^{\prime}(z)(1+\bar{x} z)^{e^{-2 i a}+1}, \quad|a|<1,|z|<1,
$$

and $F(0)=0$, then $F(z)$ is in $V_{a}^{k}$.

Proof. Let $\rho$ be a real number in the interval (0.1) and let $a$ be a complex number, $|a|<1$. For $f(z)$ in $V_{\alpha}^{k}$, define $F(z)$ by the equations

$$
F_{\rho}^{\prime}(z)=\frac{f^{\prime}(\rho \zeta)}{f^{\prime}(a)(1+a z)^{e^{-2 i a}+1}}, \quad \zeta=\frac{a+z}{1+a z}, \text { and } F_{\rho}^{\prime}(0)=0 .
$$

Then

$$
1+\frac{z F_{\rho}^{\prime \prime}(z)}{F_{\rho}^{\prime}(z)}=\left[1+\frac{\rho \zeta f^{\prime \prime}(\rho \zeta)}{f^{\prime}(\rho \zeta)}\right] \frac{\left(1-|a|^{2}\right) z}{(1+a z)(a+z)}+\frac{|a|^{2} z\left(1-e^{-2 i a}\right)+a-a z^{2} e^{-2 i a}}{(1+a z)(a+z)}
$$


Multiply both sides of (3.1) by $e^{i a}$, and then take the real part of both sides. The last term on the right-hand side of (3.1) then becomes

$$
\operatorname{Re}\left\{2|a|^{2} i \sin \alpha+(a / z) e^{i \alpha}-a z e^{i \alpha}\right\} \equiv \operatorname{Re}\{A\} .
$$

$F_{\rho}(z)$ is regular when $|z| \leq 1$. If we set $z=e^{i \theta}$, then $\operatorname{Re}\{A\}=0$. Thus from (3.1), we see that if $\left(a+e^{i \theta}\right) /\left(1+a e^{i \theta}\right)=e^{i \phi}$,

Since

$$
\begin{aligned}
\operatorname{Re}\left\{e^{i \alpha}\left[1+e^{i \theta} \frac{F_{\rho}^{\prime \prime}\left(e^{i \theta}\right)}{F_{\rho}^{\prime}\left(e^{i \theta}\right)}\right]\right\} \\
=\operatorname{Re}\left\{e^{i a}\left[1+\rho e^{i \phi} \frac{f^{\prime \prime}\left(\rho e^{i \phi}\right)}{f^{\prime}\left(\rho e^{i \phi}\right)}\right] \cdot \frac{\left(1-|a|^{2}\right) e^{i \phi}}{\left(1+a e^{i \theta}\right)\left(a+e^{i \theta}\right)}\right\}
\end{aligned}
$$

$$
d \theta=\frac{\left|a+e^{i \theta}\right|^{2}}{1-|a|^{2}} d \phi \text { and } \frac{\left|a+e^{i \theta}\right|^{2}}{\left(a+e^{i \theta}\right)} \cdot \frac{e^{i \theta}}{1+a e^{i \theta}}=1,
$$

taking absolute values and integrating (3.2) yields

$$
\begin{aligned}
& \int_{0}^{2 \pi}\left|\operatorname{Re}\left\{e^{i \alpha}\left[1+e^{i \theta} \frac{F_{\rho}^{\prime \prime}\left(e^{i \theta}\right)}{F_{\rho}^{\prime}\left(e^{i \theta}\right)}\right]\right\}\right| d \theta \\
& =\int_{0}^{2 \pi}\left|\operatorname{Re}\left\{e^{i a}\left[1+\rho e^{i \phi} \frac{f^{\prime \prime}\left(\rho e^{i \phi}\right)}{f^{\prime}\left(\rho e^{i \phi}\right)}\right]\right\}\right| d \phi \equiv I_{f}(\rho) .
\end{aligned}
$$

By hypothesis, $I_{f}(\rho) \leq k \pi \cos \alpha$, and $I_{f}$ is an increasing function of $\rho$. If we define $F(z)=\lim _{\rho \rightarrow 1} F_{\rho}(z)$, then from (3.3), it follows that $I_{F}(1)=I_{f}(\rho) \leq$ $k \pi \cos \alpha$, and $I_{F}(r) \leq \lim _{\rho \rightarrow 1} I_{F}(1)=\lim _{\rho \rightarrow 1} I_{f}(\rho)$, so $I_{F}(r) \leq k \pi \cos \alpha$ and $F \in V_{a}^{k}$.

Corollary 1. If $f(z)$ is in $V_{\alpha}^{k}$, then $f(z)$ is univalent whenever $0<\cos \alpha \leq$ $1 /(k+2)$.

Proof. Define $F(z)=z+A_{2} z^{2}+\cdots$ as in Theorem 6. Then

$$
\left|F^{\prime \prime}(0)\right|=\left|f^{\prime \prime}(z)\left(1-|a|^{2}\right) / f^{\prime}(a)-2 \bar{a} e^{-i a} \cos \alpha\right| \leq k \cos \alpha,
$$

using Theorem 5. Thus for $|z|<1$,

$$
\begin{aligned}
& \left|\frac{f^{\prime \prime}(z)}{f^{\prime}(z)}-\frac{2 z e^{-i a} \cos \alpha}{1-|z|^{2}}\right| \leq \frac{k \cos \alpha}{1-|z|^{2}}, \\
& \left|\frac{f^{\prime \prime}(z)}{f^{\prime}(z)}\right| \leq \frac{k \cos \alpha+2|z| \cos \alpha}{1-|z|^{2}}<\frac{(k+2) \cos \alpha}{1-|z|^{2}} .
\end{aligned}
$$

It is known that if $\left|f^{\prime \prime}(z) / j^{\prime}(z)\right| \leq \beta /\left(1-|z|^{2}\right)$ in $|z|<1$, for some appropriate constant $\beta$, then $f(z)$ is univalent in $E$. Recently, J. Becker [1] has proved that 
$\beta$ can be taken as at least 1 . Thus, by (3.5) and Becker's result, $f(z)$ is univalent in $E$ whenever $0<(k+2) \cos \alpha \leq 1$.

Corollary 2. If $f(z)$ is in $V_{a}^{k}$, then

$$
\ln \left[(1-|z|)^{(k / 2)-1} /(1+|z|)^{(k / 2)+1}\right] \cos a \leq \operatorname{Re}\left\{e^{i a} \ln f^{\prime}(z)\right\}
$$

$$
\leq \ln \left[(1+|z|)^{(k / 2)-1} /(1-|z|)^{(k / 2)+1}\right] \cos \alpha .
$$

These bounds are sharp.

Proof. From (3.4),

$$
\begin{aligned}
\left|\frac{e^{i a} z f^{\prime \prime}(z)}{f^{\prime}(z)}-\frac{2 \cos a|z|^{2}}{1-|z|^{2}}\right| & \leq \frac{k|z| \cos \alpha}{1-|z|^{2}} \\
\frac{2 r^{2} \cos \alpha-k r \cos \alpha}{1-r^{2}} & \leq \operatorname{Re}\left\{e^{i a} r e^{i \theta} \frac{d}{d\left(r e^{i \theta}\right)}\left[\ln f^{\prime}\left(r e^{i \theta}\right)\right]\right\} \\
& <\frac{2 r^{2} \cos \alpha+k r \cos \alpha}{1-r^{2}}
\end{aligned}
$$

and the conclusion of the corollary follows from integrating (3.7) with respect to $r$. Equality in (3.7) is attained in the upper and lower bounds when $f(z)$ is the function given in (2.7), and when $z=r$ and $z=-r$, respectively.

Corollary 3. If $f(z)$ is in $V_{a}^{k}$, then $f(z)$ maps

$$
|z|<2 /\left(k \cos \alpha+\sqrt{k^{2}} \cos ^{2} \alpha-4 \cos 2 \alpha\right)=R
$$

onto a convex domain. This result is sharp when $a=0$.

Proof. (3.4) implies

$$
\begin{aligned}
\operatorname{Re}\left\{1+\frac{z f^{\prime \prime}(z)}{f^{\prime}(z)}\right\} & =\operatorname{Re}\left\{\frac{1-z^{2}+e^{-i \alpha} \cos \alpha\left(k z+2 z^{2}\right)}{1-z^{2}}\right\} \\
& =\frac{r^{2} \cos ^{2} \alpha-k r \cos ^{2} \alpha+1}{1-r^{2}}, \text { when } z=-r
\end{aligned}
$$

Thus the bound on the radius of convexity imposed by (3.8) cannot be improved in the case $\alpha=0$, and at most can be improved only slightly (by a factor of $\cos \alpha$ in the second term) when $a \neq 0$.

Corollary 4. If $f(z)$ is in $V_{a}^{k}$, then $f(z)$ bas the property that $z f^{\prime}(z)$ is $a$ spirallike in the disk $|z|<\left(k-\sqrt{k^{2}-4}\right) / 2$. This result is sharp for all $k \geq 2$ and a such that $|\alpha|<\pi / 2$.

Proof. Let $f(z)$ belong to $V_{a}^{k}$ and recall that $z f^{\prime}(z)$ is $a$-spirallike if and only if $\operatorname{Re}\left\{e^{i a},\left[1+z f^{\prime \prime}(z) / f^{\prime}(z)\right]\right\}>0$. As in the proof of Corollary 2, 


$$
\begin{aligned}
& \operatorname{Re}\left\{e^{i a} \frac{z f^{\prime \prime}(z)}{f^{\prime}(z)}\right\} \geq 2 r^{2} \frac{\cos a-k r \cos \alpha}{1-r^{2}}, \\
& \operatorname{Re}\left\{e^{i a}\left[1+\frac{z f^{\prime \prime}(z)}{f^{\prime}(z)}\right]\right\} \geq \frac{r^{2}-k r+1}{1-r^{2}} \cdot \cos \alpha,
\end{aligned}
$$

the last expression being greater than zero for $|z|<\left(k-\sqrt{k^{2}-4}\right) / 2$. For the function defined by (2.7),

$$
\operatorname{Re}\left\{e^{i \alpha}\left[1-r \frac{f^{\prime \prime}(-r)}{f^{\prime}(-r)}\right]\right\}=\frac{r^{2}-k r+1}{1-r^{2}} \cdot \cos \alpha
$$

so the bound given above cannot be improved.

This corollary generalizes a result due to M. S. Robertson, who in [9] showed that functions with boundary rotation bounded by $k \pi$ have the property that $z f^{\prime}(z)$ is starlike in the disk $|z|<\left(k-\sqrt{k^{2}-4}\right) / 2$.

4. The Schwarzian derivative for functions in $V_{a}^{k}$.

Theorem 7. Let $f(z)$ be in $V_{a}^{k}, f(z)=z+a_{2} z^{2}+a_{3} z^{3}+\cdots$, and $\mu>2 / 3$. Then

$$
\max _{f \in V_{a}^{k}}\left|a_{3}-\mu a_{2}^{2}\right| \leq J(a, k, \mu)
$$

where

$$
J(\alpha, k, \mu)= \begin{cases}\frac{\cos \alpha}{3}\left[(3 \mu-2) \frac{k^{2} \cos \alpha}{4}+\frac{k}{2}|\sin \alpha|-\cos \alpha\right], \quad k>\frac{4}{3 \mu-2}, \\ \frac{\cos \alpha}{3}\left[(k-1) \cos \alpha+\frac{k}{2}|\sin \alpha|\right], \quad k \leq \frac{4}{3 \mu-2} .\end{cases}
$$

Proof. If $f(z)$ is in $V_{a}^{k}$, then $q(z)=1+z f^{\prime \prime}(z) / f^{\prime}(z)$ is in $Q_{a}^{k}$, and using (2.1),

$$
q(z)=e^{-i \alpha}\left[\frac{\cos \alpha}{2 \pi} \int_{0}^{2 \pi} \frac{1+z e^{i \phi}}{1-z e^{i \phi}} d \psi(\phi)+i \sin \alpha\right]
$$

where

$$
\int_{0}^{2 \pi} d \psi(\phi)=2 \pi \text { and } \int_{0}^{2 \pi}|d \psi(\phi)| \leq k \pi
$$

Let $f(z)=z+a_{2} z^{2}+\cdots$ and $q(z)=1+q_{1} z+q_{2} z^{2}+\cdots$, then $a_{2}=q_{2} / 2$ and $a_{3}=\left(q_{2}+q_{1}^{2}\right) / 6$, so $a_{3}-\mu a_{2}^{2}=\left(2 q_{2}-(3 \mu-2) q_{1}^{2}\right) / 12$.

$$
\begin{aligned}
& q_{1}=q^{\prime}(0)=\frac{e^{-i \alpha} \cos \alpha}{\pi} \int_{0}^{2 \pi} e^{i \phi} d \psi(\phi), \text { and } \\
& q_{2}=\frac{q^{\prime \prime}(0)}{2}=\frac{e^{-i \alpha} \cos \alpha}{\pi} \int_{0}^{2 \pi} e^{2 i \phi} d \psi(\phi) .
\end{aligned}
$$


We approximate $\psi(\phi)$ by a step function having nonzero jumps of $2 \pi \lambda_{j}$ at $\phi_{j}$, and we write $e^{i \phi_{j}=\epsilon_{j}}, j=1,2, \cdots, P$. Then the integrals in (4.2) become $q_{1}=$ $2 e^{-i a} \cos \alpha \sum_{j=1}^{P} \lambda_{j} \epsilon_{j}, q_{2}=2 e^{-i \alpha} \cos \alpha \Sigma_{j=1}^{P} \lambda_{j} \epsilon_{j}^{2}$. From (4.1) we obtain the conditions that

$$
\sum_{1}^{P} \lambda_{j}=1 \text { and } \sum_{1}^{P}\left|\lambda_{j}\right| \leq \frac{k}{2}
$$

$$
a_{3}-a_{2}^{2}=\frac{1}{3}\left[e^{-i \alpha} \cos \alpha \sum_{1}^{P} \lambda_{j} \epsilon_{j}^{2}-(3 \mu-2)\left(e^{-i \alpha} \cos \alpha\right)\left(\sum_{1}^{P} \lambda_{j} \epsilon_{j}\right)^{2}\right]=\frac{-e^{-2 i \alpha} T}{3} \text {. }
$$

By a rotation, we may assume that $e^{2 i a}\left(a_{3}-\mu a_{2}^{2}\right)$ is real and negative, so that $T$ will be positive.

$$
\begin{aligned}
T= & \operatorname{Re}\left\{(3 \mu-2) \cos ^{2} \alpha\left(\sum_{1}^{P} \lambda_{j} \epsilon_{j}\right)^{2}-e^{i \alpha} \cos \alpha \sum_{1}^{P} \lambda_{j} \epsilon_{j}^{2}\right\} \\
= & (3 \mu-2) \cos ^{2} \alpha\left[\left(\sum_{1}^{P} \lambda_{j} \cos \phi_{j}\right)^{2}-\left(\sum_{1}^{P} \lambda_{j} \sin \phi_{j}\right)^{2}\right] \\
& -\cos ^{2} \alpha \sum_{1}^{P} \lambda_{j}\left(\cos ^{2} \phi_{j}-\sin ^{2} \phi_{j}\right)+2 \sin \alpha \cos \alpha \sum_{1}^{P} \lambda_{j} \sin \phi_{j} \cos \phi_{j},
\end{aligned}
$$

where we have written $\epsilon_{j}=\cos \phi_{j}+i \sin \phi_{j}$. Now, $T \leq T_{0}$, where

$$
\begin{aligned}
T_{0}=(3 \mu-2) \cos ^{2} \alpha\left(\sum_{1}^{P} \lambda_{j} \cos \phi_{j}\right)^{2} \\
\quad-\cos ^{2} \alpha\left(2 \sum_{1}^{P} \lambda_{j} \cos ^{2} \phi_{j}-1\right)+|\sin \alpha| \cos \alpha \sum_{1}^{P}\left|\lambda_{j}\right| \cdot
\end{aligned}
$$

$T_{0}$ has a maximum either when the $\cos \phi_{j}$ assume values in the interior of the interval $[-1,1]$, or when they assume values at the endpoints of $[-1,1]$. Suppose the maximum occurs when $\cos \phi_{h} \neq \pm 1, b=1,2, \cdots, r \leq P$. Then possibly $\cos \phi_{j}= \pm 1$, for $b=r+1, r+2, r+3$. For these three values of $b, \sin \phi_{b}=0$. The partial derivatives with respect to the $\cos \phi_{b}$ must be zero, so from (4.4),

$$
\begin{aligned}
& 2(3 \mu-2) \cos ^{2} \alpha\left(\sum_{1}^{P} \lambda_{j} \cos \phi_{j}\right) \lambda_{b}-4 \lambda_{b} \cos ^{2} \alpha \cos \phi_{b}=0, \\
& \sum_{1}^{P} \lambda_{j} \cos \phi_{j}=\frac{2 \cos \phi_{b}}{3 \mu-2}, \quad b=1,2, \ldots, r .
\end{aligned}
$$

Substituting (4.5) into (4.4) and letting $\cos \phi$ be the common value of all the $\cos \phi_{b}$, 


$$
\begin{aligned}
T_{0} \leq & 2 \cos ^{2} \alpha \cos \phi\left(\frac{2 \cos \phi}{3 \mu-2}-\cos \phi \sum_{1}^{r} \lambda_{j}-\sum_{r+1}^{P} \lambda_{j}\right) \\
& +\cos ^{2} \alpha+|\sin \alpha| \cos \alpha \sum_{1}^{P}\left|\lambda_{j}\right| .
\end{aligned}
$$

Since (2.8) tells us that $(2 \cos \phi) /(3 \mu-2)-\cos \phi \Sigma_{1}^{r} \lambda_{j}=\Sigma_{r+1}^{P} \lambda_{j} \cos \phi_{j}$,

$$
T_{0} \leq 2 \cos ^{2} \alpha \cos \phi\left(\sum_{r+1}^{P} \lambda_{j} \cos \phi_{j}-\sum_{r+1}^{P} \lambda_{j}\right)+\cos ^{2} \alpha+|\sin \alpha| \cos \alpha \sum_{1}^{P}\left|\lambda_{j}\right| .
$$

Since (4.3) implies $\Sigma_{r+1}^{P} \lambda_{j} \cos \phi_{j} \leq \Sigma_{r+1}^{P}\left|\lambda_{j}\right| \leq k / 2-\Sigma_{1}^{r}\left|\lambda_{j}\right|$, and $\Sigma_{r+1}^{P} \lambda_{j}=$ $1-\Sigma_{1}^{r} \lambda_{j}$

$$
T_{0} \leq 2 \cos ^{2} \alpha \cos \phi\left[\frac{k}{2}-1-\sum_{1}^{r}\left(\left|\lambda_{j}\right|-\lambda_{j}\right)\right]+\cos ^{2} \alpha+\frac{k}{2}|\sin \alpha| \cos \alpha .
$$

The summation in the above inequality is positive, so

$$
T_{0} \leq(\cos \alpha)[(k-1) \cos \alpha+(k / 2)|\sin \alpha|]=T_{1}(\alpha, k, \mu) .
$$

If, on the other hand, the maximal value of $T_{0}$ occurs for $\cos \phi_{j}$ at the endpoints of $[-1,1], j=1,2, \cdots, P$, then we note in (4.4) that $2 \Sigma_{1}^{P} \lambda_{j} \cos ^{2} \phi_{j}-1=1$ whenever $\cos \phi_{j}= \pm 1$. Then

$$
T_{0} \leq \cos ^{2} \alpha\left[(3 \mu-2) k^{2} / 4-1\right]+(k / 2)|\sin \alpha| \cos \alpha=T_{2}(\alpha, k, \mu) .
$$

We note that $T_{2}(\alpha, k, \mu) \leq T_{1}(\alpha, k, \mu)$ if and only if $k \leq 4 /(3 \mu-2)$. This proves Theorem 7 .

Theorem 8. If $f(z)$ is in $V_{a}^{k}$, then

$$
|\{f, z\}| \leq 2[3 J(\alpha, k, 1)+(k+1)|\sin \alpha| \cos \alpha] /\left(1-|z|^{2}\right)^{2},
$$

where $\{f, z\}$ denotes the Schwarzian derivative of $f$ with respect to $z$ and $J(\alpha, k, \mu)$ is the functional in Theorem 7.

Proof. Let $f(z)$ belong to $V_{a}^{k}$ and define $F(z)=z+A_{2} z^{2}+\cdots$ as in Theorem 6 . Then

$$
\begin{aligned}
& 6\left(A_{3}-A_{2}^{2}\right)=\left\{\left[\frac{F^{\prime \prime}(z)}{F^{\prime}(z)}\right]^{\prime}-\left.\frac{1}{2}\left[\frac{F^{\prime \prime}(z)}{F^{\prime}(z)}\right]^{2}\right|_{z=0}\right\} \\
& =\{f, a\}\left(1-|a|^{2}\right)^{2}-a\left(1-e^{-2 i a}\right)\left[\frac{f^{\prime \prime}(a)}{f^{\prime}(a)}\left(1-|a|^{2}\right)-\frac{\bar{a}}{2}\left(1+e^{-2 i \alpha}\right)\right] . \\
& \{f, a\}\left(1-|a|^{2}\right)^{2} \\
& \quad \leq 6\left|A_{3}-A_{2}^{2}\right|+2|\sin \alpha| \cdot\left|\left(f^{\prime \prime}(a) / f^{\prime}(a)\right)\left(1-|a|^{2}\right)-\bar{a}(\cos \alpha) e^{-i a}\right| .
\end{aligned}
$$


Using (3.4), we obtain

$$
\left|\left(f^{\prime \prime}(a) / f^{\prime}(a)\right)\left(1-|a|^{2}\right)-a(\cos \alpha) e^{-i a}\right| \leq(k+1) \cos \alpha .
$$

If we replace $\left|A_{3}-A_{2}^{2}\right|$ with the result of Theorem 7, Theorem 8 follows from (4.6) and (4.7).

Corollary. Let $f(z)$ be in $V_{0}^{k}$. Then

$$
|\{f, z\}| \leq \begin{cases}\left(k^{2}-4\right) / 2\left(1-|z|^{2}\right)^{2}, & k \geq 4, \\ 2(k-1) /\left(1-|z|^{2}\right)^{2}, & 2 \leq k \leq 4 .\end{cases}
$$

When $k \geq 4$, the bound given above improves a bound found in [3], and is sharp. Setting $\alpha=0$ in (2.7), we see that the function defined by

$$
f_{0}(z)=\frac{1}{k}\left[\left(\frac{1+z}{1-z}\right)^{k / 2}-1\right]
$$

is in $V_{0}^{k}$, and

$$
\left\{f_{0}, z\right\}=1 / 2 \cdot\left(4-k^{2}\right) /\left(1-z^{2}\right)^{2} \text {. }
$$

The bound is also sharp when $k=2$, as shown in [10].

8. Univalent functions in $V_{a}^{k}$. We now make use of a fact stated without proof in $[2]$.

Theorem [Brannan]. If $f_{0}(z)$ belongs to $V_{0}^{k}$, then

$$
1+\frac{z f_{0}^{\prime \prime}(z)}{f_{0}^{\prime}(z)}=\frac{k+2}{4} p_{1}(z)-\frac{k-2}{4} p_{2}(z),
$$

where $p_{i}(0)=1, \operatorname{Re}\left\{p_{i}(z)\right\}>0 ;$ that is, $p_{i}(z)$ is in the class $P=Q_{0}^{2}$, for $i=1,2$.

Proof. Since in (2.5), $\Psi(\theta)$ has bounded variation on $[0,2 \pi]$, we may write $\Psi(\theta)=A(\theta)$, where $A(\theta)$ and $B(\theta)$ are two nonnegative increasing functions on $[0,2 \pi]$ satisfying the conditions in (2.2) and (2.3). Thus, if we set $A(\theta)=$ $((k+2) / 4) \phi_{1}(\theta), \quad B(\theta)=((k-2) / 4) \phi_{2}(\theta),(2.5)$ becomes

$$
\begin{aligned}
& 1+\frac{z f_{0}^{\prime \prime}(z)}{f_{0}^{\prime}(z)}=\frac{k+2}{4} \cdot \frac{1}{2 \pi} \int_{0}^{2 \pi} \frac{1+z e^{i \theta}}{1-z e^{i \theta}} d \phi_{1}(\theta)-\frac{k-2}{4} \cdot \frac{1}{2 \pi} \int_{0}^{2 \pi} \frac{1+z e^{i \theta}}{1-z e^{i \theta}} d \phi_{2}(\theta), \\
& 1+\frac{z f_{0}^{\prime \prime}(z)}{f_{0}^{\prime}(z)} \equiv \frac{k+2}{4} p_{1}(z)-\frac{k-2}{4} p_{2}(z) .
\end{aligned}
$$

Since $\int_{0}^{2 \pi} d \phi_{i}(\theta)=2 \pi, i=1,2$, the $p_{i}(z)$ are represented by the Herglotz-Stieltjes formula and hence are in the class $P$.

Theorem 9. Let $f_{a}(z)$ belong to $V_{a}^{k}$. Then 


$$
\frac{z f_{a}^{\prime \prime}(z)}{f_{a}^{\prime}(z)}=\left\{\frac{k+2}{4}\left[p_{1}(z)-1\right]-\frac{k-2}{4}\left[p_{2}(z)-1\right]\right\} e^{-i a} \cos \alpha,
$$

for some $p_{i}$ in $P=Q_{0}^{2}, \quad i=1,2$.

Proof. Differentiating both sides of (2.6), and dividing by $f^{\prime}(z)$, we obtain $f_{\alpha}^{\prime \prime}(z) / f_{\alpha}^{\prime}(z)=e^{-i a}(\cos \alpha) f_{0}^{\prime \prime}(z) / f_{0}^{\prime}(z)$.

$$
1+z e^{i a} \sec \alpha \frac{f_{a}^{\prime \prime}(z)}{f_{a}^{\prime}(z)}=1+\frac{z f_{0}^{\prime \prime}(z)}{f_{0}^{\prime}(z)}=\frac{k+2}{4} p_{1}(z)-\frac{k-2}{4} p_{2}(z),
$$

using Brannan's theorem as proved above.

We are now in a position to improve the bounds obtained in Theorem 7 for certain values. of $k$ and $\alpha$.

Theorem 10. Let $f(z)=z+a_{2} z^{2}+\cdots+V_{a}^{k}$. Then $\max _{f \in V_{a}^{k}}\left|a_{3}-a_{2}^{2}\right| \leq$ $L(\alpha, k)$, where

$$
\begin{aligned}
L(a, k)= & \frac{\cos \alpha}{12}\left[\frac{k^{2}-4}{2} \cos \alpha+(k+2)+(k-2) \sqrt{1+\frac{(k-2)(k+6) \cos ^{2} \alpha}{16}}\right] \\
= & \frac{\cos a}{12}\left[\frac{k^{2}-4}{2} \cos a+(k+2) \sqrt{1+\frac{(k-6)(k+2) \cos ^{2} \alpha}{16}}\right. \\
& \left.+(k-2) \sqrt{1+\frac{(k-2)(k+6) \cos ^{2} \alpha}{16}}\right], k>6 .
\end{aligned}
$$

Proof. In the proof of Theorem 7, we saw that if $f(z)=z+a_{2} z^{2}+\cdots$ is in $V_{a}^{k}$, then $q(z)=1+z f^{\prime \prime}(z) / f^{\prime}(z)=1+q_{1} z+\cdots$ is in $Q_{a}^{k}$ and $a_{3}-a_{2}^{2}=$ $\left(2 q_{2}-q_{1}^{2}\right) / 12$. Using the representation for $q(z)$ given in Theorem 9 ,

$$
\begin{aligned}
& q^{\prime}(z)=\left[\frac{k+2}{4} p_{1}^{\prime}(z)-\frac{k-2}{4} p_{2}^{\prime}(z)\right] e^{-i \alpha} \cos \alpha, \\
& q^{\prime \prime}(z)=\left[\frac{k+2}{4} p_{1}^{\prime \prime}(z)-\frac{k-2}{4} p_{2}^{\prime \prime}(z)\right] e^{-i \alpha} \cos \alpha .
\end{aligned}
$$

Since $q_{1}=q^{\prime}(0)$ and $2 q_{2}=q^{\prime \prime}(0)$,

$$
\begin{aligned}
a_{3}-a_{2}^{2}=\frac{e^{-i \alpha} \cos \alpha}{12}\left\{\frac{k+2}{4}[\right. & p_{1}^{\prime \prime}(0)-\frac{k+2}{4} e^{-i \alpha}(\cos \alpha)\left(p_{1}^{\prime}(0)\right)^{2} \\
& \left.-\frac{k-2}{4} p_{2}^{\prime \prime}(0)+\frac{k-2}{4} e^{-i \alpha}(\cos \alpha)\left(p_{2}^{\prime}(0)\right)^{2}\right] \\
& \left.+2\left[\frac{k^{2}}{16}-\frac{1}{4}\right] p_{1}^{\prime}(0) p_{2}^{\prime}(0) e^{-i \alpha} \cos \alpha\right\}
\end{aligned}
$$

The first two terms in (9.1) have the form $p^{\prime \prime}(0)-\mu\left[p^{\prime}(0)\right]^{2}$. If $p(z)=1+p_{1} z^{\prime \prime} \ldots$ is in $P$, then 


$$
p^{\prime \prime}(0)-\mu\left[p^{\prime}(0)\right]^{2}=2 p_{2}-\mu p_{1}^{2}=2\left(p_{2}-(\mu / 2) p_{1}^{2}\right)
$$

These quantities we can bound using the fact that $\left|p_{2}-(\mu / 2) p_{1}^{2}\right| \leq 2 \max \{1,1-\mu\}$, proven by Ziegler [12]. We take $\mu=(k+2 / 4) e^{-i a} \cos \alpha$ in the first term of (8.1) and $\mu=-(k-2 / 4) e^{-i \alpha} \cos \alpha$ in the second, and observe that

$$
\begin{aligned}
& \left|1-((k+2) / 4) e^{-i a} \cos \alpha\right|=\left\{1+(k-6)(k+2) \cos ^{2} \alpha / 16\right\}^{1 / 2}, \\
& \left|1+((k-2) / 4) e^{-i a} \cos \alpha\right|=\{1+(k+6)(k-2) \cos \alpha / 16\}^{1 / 2},
\end{aligned}
$$

(8.2) is greater than one when $k>6 ;$ (8.3) is greater than one when $k>2$.

Finally, modulus of the third term of (8.1) can be bounded using the fact that $\left|p_{i}^{\prime}(0)\right| \leq 2, i=1,2$. Application of the triangle inequality to (8.1) thus yields the conclusion of Theorem 10.

Theorem 11. If $f(z)$ is in $V_{a}^{k}$, then $f(z)$ is univalent whenever

$$
\begin{aligned}
& \cos \alpha\left[\frac{k^{2}-4}{4} \cos \alpha+\frac{k+2}{2}+\frac{k-2}{2} \sqrt{1+\frac{(k-2)(k+6) \cos ^{2} \alpha}{16}}\right. \\
& \cos \alpha\left[\frac{k^{2}-4}{4}+\frac{k+2}{2} \sqrt{1+\frac{(k-6)(k+2) \cos ^{2} \alpha}{16}} \quad \text { when } 2 \leq k \leq 6,\right. \\
& \left.+\frac{k+2}{2} \sqrt{1+\frac{(k-2)(k+6) \cos ^{2} \alpha}{16}}+2(k+1)|\sin \alpha|\right] \leq 2 \text {, when } k>6 .
\end{aligned}
$$

Proof. Using the bounds derived in Theorem 10 and the procedure of proof used in Theorem 8 , we find that

$$
|\{f, z\}| \leq(L(a, k)+2(k+1)|\sin \alpha| \cos \alpha) /\left(1-|z|^{2}\right)^{2},
$$

where $L(a, k)$ is the functional of Theorem 10. According to Nehari's test for univalence [6], $f$ is univalent in $E$ whenever $|\{f, z\}| \leq 2 /\left(1-|z|^{2}\right)^{2}$.

Corollary. If $f(z)$ is in $V_{a}^{2}$, then $f(z)$ is univalent whenever $0<\cos \alpha \leq x_{0}$, $.256<x_{0}<.257$, where $x_{0}$ is the positive root of $9 x^{3}+9 x^{2}+x-1=0$.

Proof. Setting $k=2$ in Theorem 11 , we see that $f(z)$ is univalent whenever $\cos \alpha[2+6|\sin \alpha|] \leq 2$. Writing $x=\cos \alpha$, the condition above is equivalent to $x+3 x \sqrt{1-x^{2}} \leq 1,9 x^{2}\left(1-x^{2}\right) \leq(1-x)^{2}, 9 x^{3}+9 x^{2}+x-1 \leq 0$.

This result agreed with the one obtained by Ziegler [12], who used a different approach. The result is the best known so far for this class.

For the case $k=2, M$. S. Robertson [10] has shown that for each a such that $\cos a>1 / 2$ there exists a function in $V_{a}^{2}$ which is not univalent in $E$. We conclude by generalizing this result. 
Theorem 12. If $k$ and a satisfy the incquality

$$
\cos \alpha\left|\frac{k^{2} \cos \alpha}{2}-2\right| \geq 6
$$

then there exist functions in $V_{a}^{k}$ which are not univalent.

Proof. Let $f_{\alpha}(z)$ be given by equation (2.7). Then

$$
\begin{aligned}
\left\{f_{\alpha}, z\right\} & =2 e^{-i \alpha}\left[\cos \alpha\left(1+k z+z^{2}\right)-1 / 2 e^{-2 i \alpha}\left(\cos ^{2} \alpha\right)\left(k^{2}+4 k z+4 z^{2}\right)\right] /\left(1-z^{2}\right)^{2}, \\
\left|\left\{f_{\alpha}, z\right\}\right|_{z=0} \mid & =\left|2 e^{-i \alpha} \cos \alpha-1 / 2 e^{-2 i \alpha} k^{2} \cos ^{2} \alpha\right| \geq \cos \alpha\left|2-\left(k^{2} \cos \alpha\right) / 2\right| .
\end{aligned}
$$

According to Nehari's test [6], any univalent function $f$ has the property that $|\{f, z\}| \leq 6 /\left(1-|z|^{2}\right)^{2}, z$ in $E$. Hence, functions (2.j) satisfying the inequalities (8.4) above are not univalent in $E$.

\section{BIBLIOGRAPHY}

1. J. Becker, Über Subordinationsketten und quasikonform fortsetzbare schlicht Functionen, Ph. D. Dissertation, Technischen Universität Berlin, 1970, 67 pp.

2. D. A. Brannan, On functions of bounded boundary rotation. I, Proc. Edinburgh Math. Soc. (2) $16(1968 / 69), 339-347$. MR 41 \#8642.

3. H. B. Coonce, A variational formula for functions of bounded boundary rotation, Ph. D. Dissertation, University of Delaware, Newark, Del., 1969, 34 pp.

4. O. Lehto, On the distortion of conformal mappings with bounded boundary rotation, Ann. Acad. Sci. Fenn. Ser. A I Math. Phys. No. 124 (1952), 14 pp. MR 14, 743.

5. K. Lowner, Untersuchen über die Verzerrung bei konformen Abbildungen Einheitskreises $|z|<1$, die durch Funktionen mit nicht verschwindender Ableitung geleifiet werden, Ber. Konigl. Sachs. Ges. Wiss. Leipzig 69 (1917), 89-106.

6. Z. Nehari, The Schwarzian derivative and schlicht functions, Bull. Amer. Math. Soc. 55 (1949), 545-551. MR 10, 696.

7. V. Paatero, Über die konforme Abbildung von Gebieten deren Rander von beschrankter Drehung sind, Ann. Acad. Sci. Fenn. Ser. A 33 (1931), 77 pp.

8. - - - Ü Über Gebiete von beschrankter Randdrchung, Ann. Acad. Sci. Fenn. Ser. A 37 (1933), $20 \mathrm{pp}$.

9. M. S. Robertson, Coefficients of functions with bounded boundary rotation, Canad. J. Math. 21 (1969), 1477-1482. MR 41 \#458.

10. - Univalent functions $f(z)$ for which $z f^{\prime}(z)$ is spirallike, Michigan Math. J. 16 (1969), 97-101. MR 39 \#5785.

11. - Variational formulae for several classes of analytic functions, Math. Z. $118(1970), 311-319$.

12. M. R. Ziegler, A class of regular functions related to univalent functions, $\mathrm{Ph} . \mathrm{D}$. Dissertation, University of Delaware, Newark, Del., 1970, 77 pp.

DEPARTMENT OF MATHEMATICS, FROSTBURG STATE COLLEGE, FROSTBURG, MARYLAND 21532

Current address: Department of Mathematics, United States Naval Academy, Annapolis, Maryland 21402 